\title{
De la planification au marché
}

La privatisation du secteur hospitalier en Inde (1947-2007)

From a Planned to a Market Economy : Privatization of the Hospital Sector in India (1947-2007)

\section{Bertrand Lefebvre}

\section{OpenEdition Journals}

Édition électronique

URL : http://journals.openedition.org/transcontinentales/693

DOI : 10.4000/transcontinentales.693

ISBN : 978-2-7351-1563-1

ISSN : 1775-397X

Éditeur

Editions de la maison des sciences de l'homme

\section{Édition imprimée}

Date de publication : 31 décembre 2007

Pagination : 39-55

ISBN : 978-2-200-92397-6

ISSN : 1950-1684

\section{Référence électronique}

Bertrand Lefebvre, « De la planification au marché », Transcontinentales [En ligne], 5 | 2007, document 3 , mis en ligne le 21 avril 2011, consulté le 07 septembre 2020. URL : http://journals.openedition.org/ transcontinentales/693 ; DOI : https://doi.org/10.4000/transcontinentales.693 


\title{
De la planification au marché
}

\author{
La privatisation du secteur hospitalier en Inde \\ (1947-2007)
}

\author{
Bertrand Lefebvre
}

L'Inde vient de fêter ses soixante ans d'indépendance. La croissance économique, le regain d'intérêt politique et économique dont bénéficie le pays, le futur statut de grande puissance qu'on lui promet, sont autant de motifs de fierté pour les élites du pays. L'état de santé des Indiens reste pourtant très en deçà de ces nouvelles ambitions. Si l'espérance de vie a plus que doublé depuis l'Indépendance, passant de 32 ans en 1947 à 66 ans en 2004, certains indicateurs de santé sont particulièrement mauvais. La tuberculose tue chaque année 500000 personnes ${ }^{1}$. Plus inquiétant encore, l'état de santé du pays stagne depuis dix ans, surtout en comparaison des progrès réalisés par certains voisins comme le Bangladesh ou le Pakistan, aux moyens humains et financiers pourtant plus limités. D'après le Rapport sur le développement humain ${ }^{2}$, l'indice de mortalité infantile était de 64 morts pour 1000 enfants âgés de moins d'un an, un chiffre supérieur à celui du Bangladesh (51 pour 1000) et à celui de la Chine (20 pour 1000). Cette moyenne nationale masque en outre d'importantes différences entre États, entre sexes, et entre zones rurales et urbaines. Le tableau 1 (page suivante) donne un aperçu de ces fortes disparités de santé, qui compliquent tout autant la mise en place d'une politique nationale de santé que les critiques qu'on peut lui adresser.

1 - Ministry of Health and Family Welfare, Annual Report 2002-2003, New Delhi, Government of India, 2003, p. 38.

Sur la nécessité d'investir dans le traitement de maladies longues comme la tuberculose, voir World Bank, World Development Report: Investing in Health, Washington, Oxford University Press, 1993.

2 - United Nations Development Programme, Human Development Report, New York, Oxford University Press, 2004.

Bertrand Lefebvre : Doctorant en géographie au sein de l'UMR IDEES, université de Rouen; boursier au Centre de sciences humaines de New Delhi (2006-2007). 
TABLEAU $1:$ L'INDICE DE MORTALITÉ INFANTILE EN INDE (2004)

\begin{tabular}{l|c} 
États / Pays & $\begin{array}{r}\text { Indice de mortalité infantile } \\
\text { (nombre de morts pour 1000 enfants } \\
\text { de moins de un an) }\end{array}$ \\
Orissa & 87 \\
Andhra Pradesh (rural) & 71 \\
Andhra Pradesh (urbain) & 35 \\
Haryana (fille) & 73 \\
Haryana (garçon) & 54 \\
Kérala & 10 \\
Rwanda & 87 \\
Malaisie & 8
\end{tabular}

Source: Human Development Report, 2004.

Si le système de soins n'est pas la réponse adéquate à tous les défis sanitaires de l'Inde, son organisation, son encadrement et son financement pèsent sur l'état de santé des Indiens. Vinay Kamat relève que lors d'une épidémie de malaria à Mumbai, la plupart des médecins du secteur privé ne suivaient pas le traitement indiqué par l'Organisation mondiale de la santé ${ }^{3}$. La compétition commerciale entre praticiens et le manque d'encadrement du secteur privé par les autorités publiques amènent à de mauvaises pratiques médicales et à la mise en danger des patients. L'accroissement de la population, l'allongement de l'espérance de vie, l'explosion des maladies dégénératives, entrainent par ailleurs une demande croissante de soins et notamment de soins hospitaliers. Le développement de certains groupes hospitaliers privés témoigne de l'existence d'un marché florissant et d'une demande sociale forte pour des soins de qualité.

En gardant à l'esprit ce contexte, nous voulons analyser ici l'expérience indienne de la privatisation des soins en général, et des soins hospitaliers en particulier. Au moment de l'Indépendance, l'Inde souhaite s'orienter vers un système de soins public accessible à tous. Comment soixante ans plus tard, le secteur privé est-il devenu la porte d'entrée privilégiée du système de soins pour le patient indien? La privatisation des soins hospitaliers a pris différentes formes en Inde. Elle ne peut se réduire à une simple expansion de l'offre de soins privée ou à une réforme de la politique publique de soins. Depuis 1991, année où l'Inde s'engage véritablement sur la voie des réformes économiques, les cercles académiques discutent des causes de la privatisation des soins en Inde. Certains pensent qu'en Inde, comme dans d'autres pays d'Amérique du Sud ou d'Afrique, la politique d'ajustement structurel préconisée par le Fonds monétaire international et la réduction des dépenses publiques de santé touchent les plus pauvres, accentuent les inégalités

3 - V.R. Kamat, «Private practitioners and their role in the resurgence of malaria in Mumbai (Bombay) and Navi Mumbai (New Bombay), India : serving the affected or aiding an epidemic?», Social Science \& Medicine, vol. 52, $\mathrm{n}^{\circ}$ 6, 2001, p. 885-909. 
d'accès aux soins et enterrent le principe fondateur d'un égal accès aux soins pour tous les Indiens ${ }^{4}$. D'autres chercheurs défendent au contraire les réformes, la rationalisation du système de soins public indien et la supériorité du marché comme moyen de réguler et de distribuer les soins ${ }^{5}$. Pour ce courant de pensée, l'Inde doit s'inspirer de l'expérience d'autres pays, notamment par la mise en place d'un partenariat public-privé (PPP) cohérent. Ces deux courants partagent au final une vision similaire de la privatisation des soins en ce qu'ils considèrent que les mêmes causes (le remboursement de la dette) produisent les mêmes effets (le contrôle des dépenses publiques) et les mêmes solutions (la privatisation des soins) dans tous les pays. Pourtant, comme le souligne John Mohan, la privatisation des soins sert de multiples intérêts et se développe à différents moments dans différents lieux et pour différentes raisons ${ }^{6}$.

Dans son étude comparée des réformes des systèmes de santé, Carolyn Tuohy suggère que la compréhension des politiques de santé et de la privatisation des soins nécessite de s'intéresser à leur base locale ${ }^{7}$. Les relations entre les médecins, les hôpitaux et les institutions locales, et la routine de ces interactions, interfèrent dans la bonne application des réformes d'ampleur nationale. La trajectoire du système de soins est aussi fonction de dynamiques locales fortes. Le contexte économique et la capacité variable à l'innovation de telle ou telle région, de même que certaines traditions locales comme l'action caritative, créent de fortes disparités territoriales dans le développement de l'offre de soins hospitaliers.

En s'inspirant de cette approche sensible aux discontinuités territoriales et en n'oubliant pas les effets structurants des différentes échelles de pouvoir (international, national, régional, local ${ }^{8}$, nous mettrons en perspective les mécanismes à l'œuvre dans la privatisation des soins hospitaliers en Inde. Dans une première partie, nous présenterons les objectifs, les réalisations et les échecs de la politique hospitalière indienne, telle qu'elle fut pensée à l'Indépendance et dans les années qui suivirent (1947-1970). Dans une deuxième partie, nous reviendrons sur l'importance du dynamisme local dans l'essor du secteur hospitalier privé (19601990). Nous présenterons ainsi le cas de l'Andhra Pradesh, un État où l'émergence de nouveaux acteurs privés et les réformes locales du système de soins ont abouti dès les années 1980 à une privatisation notable du secteur hospitalier. Cette privatisation fut par ailleurs bien documentée dès ses origines ${ }^{9}$. Enfin, dans une troisième partie, nous reviendrons sur l'impact des réformes de santé nationales dans la privatisation de l'offre hospitalière (1980-2000).

\footnotetext{
4 - Voir M. Rao (éd.), Disinvesting in Health. The World Bank's Prescriptions for Health, New Delhi, Sage, 1999.

5 - Les travaux de Peter Berman offrent un bon aperçu de ce courant. Voir P. Berman et M. E. Khan (éds), Paying for India's Health Care, New Delhi, Sage, 1993.

6 - J. Mohan, Planning, Markets and Hospitals, Londres, Routledge, 2002, p. 115.

7 - C. J. Tuohy, Accidental Logics. The Dynamics of Change in the Health Care Arena in the United States, Britain, and Canada, New York, Oxford University Press, 1999.

8 - J. Mohan, "Explaining geographies of health care : a critique», Health and Place, $\mathrm{n}^{\circ}$ 3, 1998, p. $113-124$.

9 - R. V. Baru, Private Healthcare in India : Social Characteristics and Trends, New Delhi, Sage, 1998.
} 
À l'Indépendance, le parti du Congrès, qui va longtemps gouverner, rêve d'une Inde moderne, au diapason des autres grandes nations. Comme d'autres pays fraîchement décolonisés, l'Inde veut développer un État providence, capable de promouvoir la santé de tous ses citoyens. Il existe à l'époque un large consensus sur ce choix. Avant l'Indépendance, des communistes aux grands patrons en passant par le Congrès ${ }^{10}$, tous s'accordaient sur la nécessité pour la future Inde d'avoir un État capable de dispenser des soins gratuits et accessibles à tous. Plusieurs raisons expliquent une telle unanimité. Dans les années qui suivirent la Deuxième Guerre mondiale, le modèle de l'État providence était considéré comme un succès. Les États-Unis et le Royaume-Uni avaient prouvé que l'action redistributrice de l'État pouvait entraîner création de richesse et amélioration généralisée des conditions de vie pour les populations. Dans un contexte marqué par le souvenir du tragique essor du fascisme, l'intervention de l'État dans l'économie et le domaine social semblait un moyen de protéger le capitalisme de ses démons et de consolider durablement la démocratie. Le Congrès fut aussi fortement influencé par le fabianisme, un mouvement socialiste britannique de la fin du xix siècle prônant une transformation graduelle de la société capitaliste par une planification étatique forte.

Le rapport du comité Bhore (1946) servit de base à la nouvelle politique de santé indienne. Commandité avant l'Indépendance, ce rapport pointait les défaillances du système de soins colonial : absence de politique de prévention, inégale répartition spatiale du système de soins, sous-encadrement du secteur de soins privé. Selon les recommandations de ce rapport, l'Inde devait se doter d'un système de soins entièrement public et intégré, inspiré du National Health Service (NHS) britannique. Ce tournant dirigiste par rapport au système de soins colonial a donné lieu à des tiraillements incessants dans l'orientation de la politique de santé indienne. Pour le lobby médical et le ministère de la Santé, l'expansion du système de soins était essentielle à l'amélioration de l'accès aux soins pour les Indiens. Considérant que l'état de santé des Indiens s'améliorerait plus sûrement par une meilleure alimentation, l'accès à l'eau potable et le contrôle des maladies contagieuses, la Commission du plan préférait investir dans des mesures préventives. Ce tiraillement se reflète dans l'évolution des dépenses des différents plans quinquennaux ${ }^{11}$. Si les premiers plans sont marqués par un investissement conséquent dans la formation de professionnels et dans les infrastructures, le contrôle des maladies contagieuses et l'assainissement représentent plus de la moitié des dépenses de santé (tableau 2). À la fin des années 1960, les dépenses vont s'orienter vers le planning familial au détriment des dépenses confortant le système de soins proprement dit. La part des dépenses de santé dans les différents plans quinquennaux se

10 - Voir le Bombay Plan, présenté par le patronat, le People's Plan du Parti communiste et les conclusions de la Commission nationale du Plan.

11 - Pour un compte rendu du rôle de la Commission du plan en matière de santé voir R. Jeffery, «Health planning in India 1951-1984: the role of the Planning Commission», Health Policy and Planning, vol. $1, \mathrm{n}^{\circ} 2,1986$, p. 127-137. 
réduit lentement, passant de 3,3\% au milieu des années 1950 à 1,8 \% à la fin des années 1970. Ce tassement relatif des dépenses dans le système de soins public ne sera pas sans conséquence sur la privatisation des soins.

\section{TABLEAU 2 : LES DÉPENSES dE SANTÉ DANS LES QUATRE PREMIERS PLANS QUINQUENNAUX (1951-1974)}

\begin{tabular}{l|c|c|c|c} 
Postes de dépenses (\%) & $\begin{array}{c}\text { Plan 1 } \\
(1951-1956)\end{array}$ & $\begin{array}{c}\text { Plan 2 } \\
(1956-1961)\end{array}$ & $\begin{array}{c}\text { Plan 3 } \\
(1961-1966)\end{array}$ & $\begin{array}{c}\text { Plan 4 } \\
(1969-1974)\end{array}$ \\
\hline Formation et recherche médicale & 15,4 & 16 & 16,4 & 8,5 \\
Hôpitaux et dispensaires & 17,9 & 16 & 18,1 & 14,3 \\
Système des médecines indigènes & 0,3 & 1,8 & 2,9 & 1,4 \\
Contrôle des maladies contagieuses & 16,5 & 28,4 & 20,6 & 11 \\
Planning familial & 0,5 & 1,3 & 7,9 & 27,3 \\
Eau potable et assainissement & 35 & 33,8 & 30,8 & 35,2 \\
Autres programmes & 14,4 & 2,7 & 3,3 & 2,4 \\
Dépenses de santé (millions de Rs) & 1400 & 2250 & 3415 & 11555
\end{tabular}

Source : R. Jeffery, The Politics of Health in India, Berkeley, University of California Press, 1988.

La privatisation de l'offre de soins hospitaliers est en germe dans les hôpitaux publics et dans leur crise. La double pratique publique/privée des médecins hospitaliers a été un des ferments de la privatisation des soins en Inde. Ce phénomène n'est pas spécifique à l'Inde: Metboul a montré comment, en Algérie, le clientélisme et la pratique privée ont miné de l'intérieur les hôpitaux publics ${ }^{12}$. La double pratique présente de nombreux avantages pour le médecin. L'hôpital public lui assure un revenu régulier et la sécurité de l'emploi. Au début de sa carrière, il peut s'y créer une clientèle. Le praticien peut aussi bénéficier des équipements et de l'infrastructure de l'hôpital public pour y soigner ses patients privés. L'hôpital lui procure un réseau de contacts et un statut monnayable plus tard dans le secteur privé. Les cliniques privées comptent en effet sur des consultants du secteur public pour attirer à elles un flux régulier de patients.

Contre les recommandations du comité Bhore, préconisant d'interdire aux médecins du secteur public d'exercer dans le privé et aux praticiens privés d'avoir des consultations dans les institutions publiques, le comité Mudaliar (1961) estima nécessaire d'ouvrir les hôpitaux publics à ces pratiques. Le réseau public de soins était alors en pleine expansion, et la main-d'œuvre médicale manquait. Considérant qu'une large part de médecins (40 à 70 \% selon les États et les spécialités) travaillait dans le secteur privé, et qu'il fallait mobiliser toutes les forces disponibles pour l'application des programmes de santé (vaccinations, campagnes de prévention), le comité

12 - M. Metboul, «L'introuvable césure public-privé en Algérie. De nouveaux objets en santé dans le champs de la privatisation des soins», Autrepart, $\mathrm{n}^{\circ} 29,2004$, p. 13-28. 
Mudaliar proposa que les praticiens privés reçoivent le statut de consultants dans les hôpitaux publics et soient autorisés à y opérer leurs patients. Le brouillage de la frontière entre public et privé n'était pas nouveau dans les hôpitaux en Inde. Déjà, la East India Company autorisait ses chirurgiens exercer une activité privée très rémunératrice. Cet usage perdura sous le British Raj, après 1857, et les médecins des hôpitaux publics eurent de fait un avantage concurrentiel décisif sur les médecins du privé : la sécurité de l'emploi leur permettait de pratiquer des tarifs inférieurs et leur présence dans les hôpitaux publics leur donnait accès à un large panel de patients et de thérapies. Au tournant de la Grande Dépression, et afin d'encourager la pratique privée dans la Présidence de Madras, il fut même décidé d'affilier de nombreux médecins privés sans emplois aux hôpitaux publics pour leur permettre de rivaliser avec les médecins du secteur public ${ }^{13}$.

Le retournement de 1961 peut être considéré comme une victoire du lobby médical et comme un retour à la situation coloniale. Lorsque le comité Jungawalla (1967) proposa de revenir aux principes défendus par le comité Bhore, il évita toute posture radicale. L'interdiction de la pratique privée se limita à quelques catégories, celle des enseignants et des chercheurs, puis celle des médecins exerçant un poste de responsabilité dans une structure publique. La santé et le système de soins relevant aussi de la compétence des États, certains gouvernements locaux essayèrent de mettre fin à la double pratique publique et privée. L'Andhra Pradesh $(1968,1984)$, l'Uttar Pradesh (1975), le Bihar (1986) interdirent cette pratique. Les grèves, les démissions de médecins seniors, l'insertion des médecins dans les réseaux politiques, et la menace d'un chaos complet dans les hôpitaux publics eurent rapidement raison de ces lois.

La crise des hôpitaux publics trouve aussi ses racines dans le tassement des investissements publics. Alors que les années 1950-1960 avaient été marquées par une expansion importante de l'infrastructure hospitalière publique, les décennies suivantes ont vu les dotations budgétaires se détourner vers le planning familial et le contrôle reproductif, secteurs jugés prioritaires. Cette crise de l'investissement public eut des conséquences sur les hôpitaux publics. Les conditions de travail se dégradèrent. Les hôpitaux vieillirent sans bénéficier d'investissements conséquents dans leur rénovation ou dans leur équipement, alors même que le nombre de patients à traiter augmentait. La hausse du nombre de patients trouvait son origine dans la faillite du système de soins hiérarchisé mis en place après l'Indépendance. Les postes de santé primaire, sous-équipés et en nombre insuffisant dans certaines régions, ne pouvaient jouer leur rôle de porte d'entrée d'un système de soins supposé être accessible à tous. L'absentéisme des médecins et le clientélisme n'y arrangeaient rien. Le système de référencement qui devait permettre une gestion rationnelle des flux de patients était en échec, et les hôpitaux publics se retrouvèrent submergés. Dans les villes, les patients ignoraient l'existence des dispensaires publics et préféraient aller directement dans un grand hôpital public. La politique

13 - V.R. Muraleedharan, «Rural health care in Madras Presidency : 1919-1939», Indian Economic \& Social History Review, $\mathrm{n}^{\circ} 24,1987$, p. 323-334. 
ambitieuse consistant à développer de manière concomitante un réseau de soins tertiaires de qualité et une infrastructure de soins primaires se retourna contre le système dans son ensemble et contribua à son effondrement. Dans une étude sur les médecins du All India Institute of Medical Science (AIIMS) de New Delhi, institution nationale de référence, Rama Baru montre comment la dégradation des conditions de travail affecta le sens du devoir des médecins et leur attachement à l'institution $^{14}$. L'AIIMS était une institution d'excellence, offrant de bonnes conditions de travail et de recherche aux médecins. Elle était un des temples de la modernité chers à Nehru. Un sentiment de lassitude gagna pourtant le corps médical. Progressivement de plus en plus de médecins quittèrent l'AIIMS pour l'étranger. Les nouvelles générations de médecins eurent des aspirations plus individuelles, plus carriéristes.

Le sous-investissement créa aussi des distorsions dans le marché de l'emploi médical. Au cours des années 1950 et 1960, alors que le pays manquait de médecins, de nombreuses facultés de médecine furent créées. À partir des années 1970 , le système de soins public dans son ensemble ne put absorber l'augmentation du nombre de diplômés, faute de nouveaux postes. De plus en plus de médecins n'eurent d'autres choix que d'ouvrir un cabinet privé pour travailler ou de s'expatrier. Dans les années 1970 la planification se trouva en échec. Elle n'arrivait plus à ajuster le rythme de formation de professionnels au nombre de postes disponibles. À ce décalage en termes d'emplois s'ajoutaient aussi les effets de la distorsion entre la médecine biomédicale et les besoins de base de la société. Dans une Inde très majoritairement rurale, les besoins de santé reposaient plus sur l'amélioration de l'alimentation et des infrastructures (l'accès à l'eau potable en particulier) et sur la lutte contre les maladies vectorielles (malaria, dengue). Mais les intérêts de certains groupes professionnels et sociaux passèrent avant l'intérêt général.

\section{L'entrepreneur, le "NRI» et le patient... acteurs de la privatisation (1960-1990)}

Dans certains États du sud de l'Inde $^{15}$, cette distorsion entre la formation et le système de soins public eut d'importantes répercussions, d'abord à une échelle locale puis pour l'ensemble du système de soins indien. Dès la période coloniale, le sud de l'Inde était à la pointe de la médecine biomédicale par rapport aux autres régions. Parfois sous l'impulsion des activités missionnaires, les dispensaires et les hôpitaux se développèrent. Parallèlement, le nombre de facultés de médecine augmenta. En 2004 encore, plus de 40 \% des facultés de médecine indiennes se trouvaient dans ces États qui ne comptent que $20 \%$ de la population indienne. C'est en raison de cet héritage fécond et de certaines décisions politiques que le secteur hospitalier privé s'est développé rapidement dans le Sud et particulièrement en Andhra Pradesh. Le poids de l'offre hospitalière privée, tout autant que le recours

14 - R.V. Baru, "Commercialization and the public sector in India : implications for values and aspirations", in M. Mackintosh et M. Koivusalo (éds), Commercialization of Health Care. Global and Local Dynamics and Policy Responses, Londres, Palgrave Macmillan, 2005, p. 101-116.

15 - Andhra Pradesh, Tamil Nadu, Kérala, Karnataka et Pondichéry. 
à cette offre, en firent un des États en pointe de la privatisation des soins hospitaliers ${ }^{16}$. À travers l'exemple de l'Andhra Pradesh, on peut comprendre comment la privatisation des soins hospitaliers prend sa source dans des dimensions sociales, économiques et politiques locales.

La multiplication des cliniques et des hôpitaux privés en Andhra Pradesh est symptomatique d'un long changement social en milieu rural. Beaucoup de brahmanes abandonnèrent l'agriculture sous l'Empire britannique. Devenus urbains et travaillant pour l'administration coloniale, ils revendirent leurs terres à des castes paysannes. Une nouvelle classe d'agriculteurs émergea dans les campagnes de l'Andhra Pradesh : les Reddy, les Khamma, les Naidu, les Raju. Ces castes ont réussi à intensifier les cultures et à dégager ainsi des profits supplémentaires de leurs activités agricoles. Cette nouvelle classe de capitalistes ruraux a alors réinvesti ses profits dans d'autres secteurs économiques: le commerce, le bâtiment, l'immobilier, la petite industrie. Cette stratégie de diversification ne s'explique pas uniquement par un esprit d'entreprise développé. Le risque latent d'une nouvelle réforme agraire redistribuant les terres aux plus pauvres menaçait la position de ces castes dominantes du système agricole ${ }^{17}$. Même si la «révolution verte» rendait l'agriculture lucrative, la possibilité d'une réforme agraire ne les incitait pas à investir dans l'expansion des propriétés foncières, mais plutôt à chercher une porte de sortie. L'investissement dans l'éducation de leurs enfants fut une de ces stratégies d'esquive. Dans certains villages, les Khamma firent des choix risqués, en vendant une partie de leurs terres pour financer les études de leurs enfants dans des écoles d'ingénieurs ou des facultés de médecine. Par le passé, la paysannerie de l'Andhra côtier avait déjà investi dans la construction de logements étudiants et le financement de bourses pour encourager les études de leurs enfants. Leur poids économique accru et leurs réseaux politiques leur ouvrirent de nouvelles opportunités. Par le biais des pressions politiques, et en accordant des donations aux facultés, ils obtinrent une augmentation du nombre d'étudiants, voire la création de facultés de médecine. Lorsque, devenus médecins, leurs enfants créèrent des cliniques ou des hôpitaux, leur famille apporta un soutien financier. La nationalisation des banques de 1969 leur donna aussi un accès plus facile au crédit. Les prêts se démocratisèrent et touchèrent de nouveaux secteurs de l'économie: l'agriculture, la petite industrie, la santé, l'éducation. Rama Baru rappelle l'importance de ces deux sources de financement dans l'essor des cliniques privées à Hyderabad ${ }^{18}$, où de nombreux promoteurs de ces établissements médicaux sont issus de ces castes d'agriculteurs (Reddy, Khamma, Raju). Baru a constaté également que plus la taille de la clinique est importante, plus on trouve de promoteurs issus de riches familles d'agriculteurs ou de grands propriétaires terriens.

16 - Dès les années 1980, le recours au secteur privé pour les hospitalisations y est le plus élevé en Inde (National Statistical Survey Organisation, Socio-Economic Survey, Round 42, New Delhi, Government of India, 1987).

17 - G. Omvedt, "Capitalist agriculture and rural classes in India», Economic and Political Weekly, vol. 16, n52, 1981, p. 140-159.

18 - R. V. Baru, op. cit., 1998. 
Dans un État comme l'Andhra Pradesh, l'interdiction de la pratique privée faite aux médecins du secteur public eut des répercussions inattendues. Au cours des dixhuit mois qui suivirent la première interdiction de 1968, de nombreux médecins seniors démissionnèrent de leur poste dans les hôpitaux publics, et rejoignirent le secteur privé ou quittèrent l'Andhra Pradesh. En 1983, le parti régionaliste nouvellement arrivé au pouvoir, le Telugu Desam, réimposa cette interdiction qui dura six années jusqu'au retour aux commandes du parti du Congrès en 1989. Entre-temps, des médecins seniors et juniors quittèrent à nouveau les hôpitaux publics. Toujours d'après l'enquête de Rama Baru ${ }^{19}$, une majorité des propriétaires de cliniques privées ayant précédemment pratiqué dans le secteur public ont rejoint le secteur privé à la suite des interdictions de cumul.

Les médecins NRI ${ }^{20}$ furent d'autres acteurs importants de la privatisation des soins hospitaliers en Inde, en Andhra Pradesh en particulier. À partir des années 1960, un nombre croissant de médecins indiens quitta le pays. Comme nous l'avons précédemment souligné un tel mouvement trouva une partie de son origine dans l'inadéquation entre la formation médicale et les besoins de soins en Inde. Les meilleures conditions de travail, les meilleurs salaires, l'opportunité de faire de la recherche médicale de haut niveau, sont des facteurs tout aussi importants dans le choix de l'étranger. Les États-Unis au premier chef, mais aussi le Royaume-Uni et les pétromonarchies du Moyen-Orient sont toujours des destinations privilégiées. Les départs concernent surtout les étudiants des meilleures facultés de médecine comme l'AIIMS ou la faculté de médecine de Baroda. Environ 38000 médecins $\mathrm{d}^{\prime}$ origine indienne pratiqueraient aujourd'hui aux États-Unis ${ }^{21}$. En effet dans les années 1960 aux États-Unis, les programmes Medicare et Medicaid permirent aux pauvres et aux personnes âgées d'avoir accès au système de soins. Pour répondre à cette croissance de la demande, le gouvernement fédéral américain soutint la création de nombreuses cliniques et hôpitaux privés par une politique d'exemption fiscale et de subventions. Le manque de main-d'œuvre médicale se fit alors durement sentir. Des investissements dans la formation du personnel médical et le relâchement des règles migratoires furent décidés. Toutefois la crise économique des années 1970 toucha durement le secteur hospitalier. La volonté des assureurs de mieux maîtriser les dépenses de santé entraîna un mouvement de concentration du secteur. Des hôpitaux fermèrent ou réorientèrent leur activité vers les branches les plus rentables. Lors du premier mandat du président Reagan, les conditions d'éligibilité au programme Medicaid se durcirent. Certaines chaînes hospitalières connurent d'importantes difficultés financières. Dans ce contexte morose, les investissements opérés lors de la décennie précédente en faveur de la formation portèrent leurs fruits au plus mauvais moment. Entre 1965 et 1980, le nombre de nouveaux médecins diplômés passa de 7409 à $15135^{22}$. Avec le

19 - R. V. Baru, op. cit., 1998.

20 - NRI pour Non Resident Indian. Cet acronyme désigne, dans la diaspora indienne, ceux qui ont gardé la nationalité indienne.

21 - S. Raymer, «Indian doctors help fill US health care needs», Yale Global, 16 février 2004.

22 - P. Starr, The Social Transformation of American Medicine, New York, Basic Books, 1982, p. 421. 
maintien d'une forte immigration de praticiens étrangers, le marché américain des soins parut saturé.

Certains praticiens NRI partis aux États-Unis dès les années 1960 commencèrent à revenir en Inde vers le milieu des années 1970. Ces pionniers du retour le firent pour des raisons très personnelles, parfois teintées de patriotisme. Ces figures discursives présentées aux médias ne doivent pas faire oublier que les NRI de l'Andhra Pradesh sont souvent issus de la classe entrepreneuriale précédemment décrite. Le père de Prathap Reddy, le fondateur d'Apollo Hospitals, était un grand propriétaire terrien du sud de l'Andhra Pradesh. Tirant profit de leur réseau familial, du prestige conféré par leur pratique à l'étranger, ces médecins réussirent à développer leur activité. En mobilisant leur réseau professionnel aux États-Unis, certains médecins NRI purent ainsi ouvrir des cliniques privées, équipées des dernières innovations en matière de technologies médicales. Tous les médecins NRI ne revinrent pas en Inde. Beaucoup préférèrent simplement y investir. Lorsqu'en 1983, Prathap Reddy ouvrit son premier hôpital à Chennai, 40 \% de l'investissement initial venait de collègues NRI restés aux États-Unis. Certains des plus grands hôpitaux privés d'Hyderabad sont le fait de NRI (Medwin Hospital, Mediciti).

L'ascension sociale de certains groupes et la circulation migratoire ont pesé dans la privatisation accélérée des soins hospitaliers en Andhra Pradesh. Ailleurs en Inde, fût-ce plus tardivement, on peut retrouver tout ou partie de ces éléments constitutifs de la privatisation. Si l'émergence de l'offre de soins privée a certainement favorisé la privatisation des soins hospitaliers, la demande de soins y a également contribué.

TABLEAU 3 : Les CAS D'hosPITALISATION DANS LE SECTEUR PUBLIC ET LE SECTEUR PRIVÉ EN INDE, EN POURCENTAGE (1986-2004)

\begin{tabular}{l|r|r|r|r|r|r}
$\begin{array}{r}\text { Cas } \\
\text { d'hospitalisation } \\
\text { (\%) }\end{array}$ & \multicolumn{3}{|c|}{ Zone rurale } & \multicolumn{3}{c|}{ Zone urbaine } \\
\begin{tabular}{l|c|c|c|c|c|} 
Lieux \\
d'hospitalisation
\end{tabular} & $1986-1987$ & $1995-1996$ & 2004 & $1986-1987$ & $1995-1996$ & 2004 \\
Hôpitaux publics & 59,7 & 43,8 & 41,7 & 60,3 & 43,1 & 38,2 \\
Hôpitaux privés & 40,3 & 56,2 & 58,3 & 39,7 & 56,9 & 61,8 \\
\hline Total & 100 & 100 & 100 & 100 & 100 & 100
\end{tabular}

Sources : National Statistical Survey Organisation, Socio-Economic Surveys : Round 42 (1987), Round 52 (1996), Round 60 (2004).

Le tableau 3 récapitule l'évolution de l'utilisation des soins hospitaliers de 1986 à 2004 en Inde. En 1986, autour de 60 \% des hospitalisations se faisaient dans le secteur public. En l'espace de dix ans, la tendance s'inverse, la majorité des hospitalisations ayant désormais lieu dans le secteur privé (56\% en zone rurale, $57 \%$ en zone urbaine). Le retournement est particulièrement spectaculaire en zone urbaine et s'est poursuivi de manière ralentie entre 1996 et 2004. La logique enclenchée 
au cours des années 1970 donne sa pleine mesure à partir des années 1980. Le manque d'investissement dans le secteur public entraîne une détérioration de la qualité du service : les patients ont l'impression d'être mieux considérés et mieux traités dans le secteur privé. L'embryon de classe moyenne et supérieure qui continuait à venir dans les hôpitaux publics a déserté le premier ces infrastructures. L'absentéisme, le détournement des patients du secteur public vers les cabinets de pratique privée affectèrent la notion même de service public. Dans des États comme le Bihar, où le secteur hospitalier public connaît une débâcle financière sans précédent, l'hospitalisation en secteur privé est ainsi passée en zone rurale de $29 \%$ à $86 \%$ entre 1986 et $2004^{23}$. Finalement, l'essor de la société de consommation à partir des années 1980 et les nouvelles aspirations de la classe moyenne indienne vont aussi toucher le secteur de la santé. L'offre privée se présente désormais avec un spectre complet de thérapies, un souci renouvelé du confort du patient et de meilleures infrastructures que le secteur public. Même si le choix du secteur privé n'a pas seulement été le fait des classes moyennes et supérieures ${ }^{24}$, on peut lire l'essor des hôpitaux privés et de leur utilisation au cours des années 1980 comme un mouvement de sécession de la nouvelle classe moyenne. L'Inde n'est jamais devenue l'État providence pensé à l'Indépendance. Les différents programmes nationaux d'assurance et de couverture sociale, comme le Central Government Health Scheme, n'ont bénéficié qu'à une minorité de privilégiés. Au fur et à mesure, les grandes entreprises publiques ont fait des donations, notamment d'équipement médical, aux hôpitaux privés, pour obtenir de meilleurs tarifs pour leur personnel. La base sociale qui aurait pu préserver et encourager le développement de l'État providence ne fut jamais assez large.

\section{Le temps des réformes:}

vers le paradoxal retour de l'État? (1980-2000)

Contrairement à d'autres pays comme le Chili ${ }^{25}$ ou la Nouvelle-Zélande ${ }^{26}$, la privatisation des soins hospitaliers résulte donc moins d'une réorientation des politiques de santé à l'échelle nationale, que d'une transformation endogène et lente du système de soins. Au début des années 1980, le discours est néanmoins à la réforme du système de soins et à la nécessaire reconnaissance du secteur privé. Alors que la crise des finances publiques se fait de plus en plus sentir et que le modèle mis en place au cours des années 1950 est à bout de souffle, incapable d'atteindre son objectif universaliste, un nouveau modèle doit être inventé.

23 - Source: National Statistical Survey Organisation, Socio-Economic Surveys, Round 42 (1987), Round 60 (2004).

24 - C.A. K. Yesudian, "Behaviour of the private sector in the health market of Bombay", Health Policy and Planning, vol. 9, $\mathrm{n}^{\circ} 1,1994$, p. 72-80.

25 - E. Miranda, J. L. Scarpaci et I. Irarràzaval, "A decade of HMOs in Chile : market behavior, consumer choice and the state», Health and Place, vol. 1, ${ }^{\circ} 1,1995$, p. 51-59.

26 - T. Ashton, N. Mays et N. Devlin, "Continuity through change: the rhetoric and reality of health reform in New Zealand», Social Science \& Medicine, ${ }^{\circ}$ 61, 2005, p. 253-262. 
Dès 1982, le document cadre National Health Policy indique la voie à suivre ${ }^{27}$. Pour la première fois, un document gouvernemental reconnaît non seulement l'importance du secteur privé dans la délivrance de soins, mais surtout l'importance pour la puissance publique de soutenir le développement du secteur privé afin de remplir l'objectif de la "santé pour tous en 2000 ». Un tel changement de direction se traduit en des termes politiques très concrets. Le sixième plan quinquennal (1980-1985) reconnaît le rôle joué par le secteur privé dans l'offre de soins. Le secteur de la santé acquiert le statut d'industrie, ce qui permet aux acteurs privés du secteur d'accéder aux grandes institutions financières publiques comme l'Industrial Development Bank of India. L'obtention de prêts à des taux intéressants et l'allègement des taxes douanières sur l'importation de matériel médical de haute technologie (de $107 \%$ à $40 \%$ ) vont encourager le développement de nouvelles cliniques. C'est à cette époque que certains groupes hospitaliers privés comme Apollo Hospitals s'étendent dans le sud de l'Inde. Jouant de leurs réseaux politiques, certains acteurs économiques investissent et spéculent sur le développement du marché des soins hospitaliers. Certaines autorités locales allouent gratuitement ou à des prix subventionnés des terrains pour la construction de nouveaux hôpitaux privés. Dès les années 1980, un embryon de partenariat public-privé existe donc. Selon certaines estimations établies en 2004, 65 milliards de roupies ont été distribués depuis 1983 à travers ces exemptions fiscales et ces subventions ${ }^{28}$.

À la suite de la crise financière du printemps 1991, l'Inde recourt aux organismes financiers internationaux. Une politique d'ajustement structurel visant au remboursement de la dette est lancée. La Banque mondiale reconnaît elle-même que ces ajustements se traduisent par la réduction des dépenses publiques de santé, notamment dans le secteur des soins tertiaires ${ }^{29}$. Ce changement de direction macroéconomique ne sera donc pas sans conséquences sur le système de soins indien et sur le système hospitalier public. L'investissement public dans la santé diminue un peu plus. Aux termes de la Constitution, la santé fait partie de la liste des compétences partagées entre le gouvernement central et les États. Si l'investissement central s'est plus ou moins maintenu autour de 1,3\% du budget total, celui des États s'est effrité de $7 \%$ à 5,5\% ${ }^{30}$. Le gouvernement central est en grande partie responsable de cette réduction dans la mesure où, par des mécanismes de péréquation et de soutien à certains programmes prioritaires, il finançait à hauteur de $60 \%$ le budget santé des États. Cette part chuta à $40 \%$ en 2001. Sous couvert de décentralisation, l'ajustement structurel déplaça la charge financière de la santé

27 - «Afin de réduire les dépenses gouvernementales et d'utiliser pleinement les ressources disponibles, des programmes pourront être définis en fonction des besoins et des potentiels locaux, pour encourager des acteurs non gouvernementaux à établir des centres de soins, et pour offrir un soutien logistique, financier et technique aux instances prêtes à intervenir dans le domaine de la santé.» (Government of India, National Health Policy, New Delhi, Government of India, 1984, p. 8)

28 - National Commission on Macroeconomics and Health, Financing and Delivery of Health Care Services, New Delhi, Government of India, 2005, p. 53.

29 - «La reduction des dépenses publiques étant essentielle à un programme d'ajustement, les dépenses de santé doivent être diminuées.» (World Bank, op. cit., 1993, p. 45)

30 - Ministry of Health and Family Welfare, National Health Policy, New Delhi, Government of India, 2002. 
vers les États. Parfois confrontés à des crises fiscales importantes, les États les moins riches ont gravement souffert de cette politique, obligés qu'ils étaient de mobiliser leurs ressources pour payer en priorité les salaires au détriment d'autres postes financiers (équipement médical, médicaments) ${ }^{31}$. L'investissement dans le système hospitalier public chuta lourdement, passant de $25 \%$ du budget santé du gouvernement central en 1991 à moins de $6 \%$ en 2001. L'État indien mettait en pratique l'argument de la Banque mondiale selon laquelle l'État devait se désengager des soins hospitaliers tertiaires.

Pour compenser ce recul de l'investissement public et sauver les apparences, la politique de soutien au secteur privé se poursuivit, avec l'aide de la Banque mondiale. Considérant le secteur privé comme étant plus efficace en raison du jeu de la concurrence ${ }^{32}$, la Banque mondiale soutint directement le développement de certains hôpitaux privés. En octroyant un prêt de 134 millions de dollars à l'État du Maharashtra, elle s'assura que $5 \%$ de cette somme serait allouée à la construction d'un hôpital privé à Mumbai, géré par un groupe hospitalier privé ${ }^{33}$. Sa branche dévolue au secteur privé, l'International Finance Corporation, soutint aussi, par l'intermédiaire de prêts, le développement de certains groupes hospitaliers privés. L'État de Delhi est allé jusqu'à prendre une participation de $26 \%$ dans le consortium basé à l'île Maurice contrôlant l'Indraprastha Apollo Hospital. En contrepartie, les nouveaux hôpitaux privés doivent réserver $25 \%$ de leurs lits aux plus démunis ou organiser des camps de soins gratuits dans les bidonvilles ou dans les villages. Ce furent les grandes heures du partenariat public-privé où, sous l'égide de la Banque mondiale, l'efficacité et la compétence des hôpitaux privés devaient se mettre au service de la santé publique. Cette politique n'a pas donné les résultats escomptés, les hôpitaux privés ne respectant pas leurs engagements ${ }^{34}$. En fait, dès le début des années 1990, à la suite des premières tentatives de partenariat public-privé des années 1980, la presse se faisait l'écho de tels manquements. Le partenariat publicprivé possédait certainement une dimension expérimentale dont les conséquences ont été sous-estimées par ses promoteurs. On a demandé à une administration d'inventer en quelques années une culture du partenariat et de la négociation. Certains contrats furent négociés à la hâte et se révélèrent inapplicables, la définition des patients pauvres et des missions assignées aux hôpitaux privés restant confuse. Un patient pauvre à Delhi doit obtenir une lettre de recommandation du Gouvernement de Delhi, ce qui nécessite de bénéficier d'un patronage politique ou de payer un dessous-de-table. La vérification de l'application d'une telle norme se révèle lourde et fastidieuse. Sous couvert d'anonymat, certains docteurs parlent de ramassage de pauvres avant la visite des délégations du gouvernement. Il existe rarement des clauses de rétorsion contre les hôpitaux ne respectant pas les normes.

31 - V. Sevalraju et al., Trends in Public Spending on Health in India, New Delhi, National Institute of Public Finance and Policy, 2001.

32 - World Bank, op. cit., 1993, p. 131.

33 - G. Yamey, "World Bank funds private hospitals in India», British Medical Journal, vol.32, 3 février 2001

34 -B. Purohit, "Private initiatives and policy options : recent health system experience in India", Health Policy and Planning, vol. 16, n ${ }^{\circ} 1,2001$, p. 87-97. 
Certains accords, notamment dans le cas de reprise d'hôpitaux publics, sont à l'avantage des acteurs privés, le risque de pertes financières étant couvert par le gouvernement. Le partenariat public-privé a surtout permis à certains groupes d'investir dans le secteur de la santé en limitant les risques et en bénéficiant de généreuses exemptions d'impôts. Certains hôpitaux privés jouent d'ailleurs habilement de leur statut pour obtenir plus d'exonérations fiscales, en faisant enregistrer leur hôpital comme centre de recherche ou comme société caritative.

Plutôt que d'engager une réforme lourde et politiquement risquée du système hospitalier public, l'État a fait le choix de la facilité en orientant les réformes vers le partenariat public-privé, pour le plus grand profit de certains groupes hospitaliers privés. Plus tard, au milieu des années 1990, une réforme ciblant les hôpitaux secondaires fut finalement lancée. L'intervention de la Banque mondiale fut déterminante et se traduisit par un soutien financier dans plusieurs États comme le Karnataka, le Bengale occidental, l'Uttar Pradesh. Les États appliquèrent différentes recettes qui devaient permettre d'assurer des soins de qualité à un coût maîtrisé : externalisation et contractualisation des services non médicaux (blanchisserie, repas, nettoyage) en premier lieu et plus tard des diagnostics (scanner, rayons X) ; contractualisation des infirmières, des médecins, des techniciens cliniques; introduction du principe de l'usager-payeur, hausse des tarifs d'hospitalisation. Cette corporatisation des hôpitaux publics connut un succès mitigé. Au lieu de repenser le système hospitalier dans son ensemble, on s'attaqua à un seul niveau de la pyramide des soins publics. Le principe usager-payeur se révéla contre-productif. Les patients les plus pauvres se retrouvèrent un peu plus exclus du système hospitalier. Les maigres fonds générés (2-3\% du budget d'un hôpital) par ce système ne furent pas forcément dépensés, et servirent plus souvent à compenser de nouvelles baisses de l'allocation financière aux hôpitaux publics comme en Andhra Pradesh et au Maharashtra.

Au total, ces vingt dernières années de réformes d'inspiration néolibérale ont été de marquées par la rencontre paradoxale de l'État et du secteur hospitalier privé. La croissance anarchique de ce secteur n'avait jamais préoccupé la puissance publique, malgré les abus et les mauvaises pratiques médicales et commerciales dont les patients ont été les premières victimes. Une meilleure régulation du secteur hospitalier privé, réclamée par certains groupes hospitaliers et par un nombre croissant de patients, rend aujourd'hui l'État plus incontournable que jamais.

\section{Conclusion}

Comme dans d'autres pays, le temps où l'État indien devait assurer seul ou presque la délivrance de soins hospitaliers semble bien révolu. Désormais sa mission semble s'orienter de plus en plus vers le financement et la régulation du secteur hospitalier privé. Ce changement de rôle ne va pas sans poser de problèmes, dans un pays où les disparités territoriales dans la répartition du système de soins et les inégalités des niveaux de santé au sein de la population restent énormes. On peut s'interroger sur la capacité des pouvoirs publics à assurer cette mission de régulation du secteur hospitalier privé au vu de l'échec des tentatives passées de 
régulation. Dès les années 1950, des textes législatifs comme le Delhi Nursing Home Act ou le Bombay Act posaient un certain nombre de normes sanitaires et de sécurité à l'ouverture et au fonctionnement de cliniques et d'hôpitaux privés. À Delhi, une minorité de cliniques et d'hôpitaux privés sont en conformité avec le Delhi Nursing Home Act. En 2003, une enquête de la direction des services de santé de l'État de Delhi révéla que $80 \%$ des cliniques privées, soit 1603 établissements, n'étaient pas enregistrées et ne répondaient pas aux cahiers des charges du Delhi Nursing Home Act, deux démarches pourtant obligatoires. En 2004, 800 cliniques étaient prêtes à entamer les démarches nécessaires à leur régularisation. En 2007, seules 500 d'entre elles étaient effectivement régularisées. L'équipe chargée du contrôle du secteur hospitalier à Delhi est composée de deux médecins et d'une poignée d'enquêteurs. Avec des moyens humains aussi limités, il semble difficile d'envisager un contrôle efficace des pratiques médicales et des conditions sanitaires de l'offre hospitalière privée. Par ailleurs, des hôpitaux privés bien en vue et ayant reçu des subsides des pouvoirs publics pour soigner les pauvres ont réussi jusqu'à présent à éviter des sanctions malgré les entorses régulières à leurs engagements ${ }^{35}$.

Une commission du ministère de la Santé indien a été créée pour mettre en place un système national d'accréditation des hôpitaux privés. Des licences seraient distribuées aux cliniques selon des critères de sécurité, d'équipement, de qualification du personnel et de taille de l'établissement. Les licences seraient valables trois ans et reconductibles après évaluation. Si un tel système se justifie à bien des égards pour lutter contre l'incurie et les mauvaises pratiques d'une partie du secteur de soins privé, il sert aussi d'autres intérêts et constitue une revendication ancienne des groupes hospitaliers privés. Dans un secteur aussi peu réglementé et aussi dispersé, la visibilité et la pénétration de ces groupes restent lentes. La mise en place d'un tel système pourrait accélérer leur développement. Certaines cliniques se verraient contraintes de fermer ou de se mettre à la recherche de partenaires financiers pour investir dans la mise aux normes de leur infrastructure. Certains représentants des groupes hospitaliers privés espèrent voir, d'ici dix ans, quelques groupes dominer le marché des soins hospitaliers, les cliniques privées de niveau secondaire ayant disparu ou s'étant intégrées dans les réseaux de ces groupes. Le lobby des médecins et leur entregent local devraient s'efforcer de ralentir une telle concentration. Il sera intéressant de voir comment les autorités publiques réagiront à la pression politique lorsque le couperet de l'accréditation tombera sur plusieurs cliniques. Si l'État reste incontournable dans le secteur hospitalier, les fondements d'une telle position semblent bien éloignés des objectifs de santé publique. L'État indien est-il au service de la population indienne, ou au service des intérêts financiers des grands groupes hospitaliers privés?

L'auteur tient à remercier Rama Baru, Venkat Raman, S. K. Rao, Alain Vaguet et Rémy Delage pour les échanges qui ont nourri la rédaction de ce texte.

35 - Public Account Committee, Allotment of Land to Private Hospitals and Dispensaries by Delhi Development Authority, New Delhi, Lok Sabha, 2005. 


\section{Bibliographie}

- Ashton T., N. Mays et N. Devlin, «Continuity through change : the rhetoric and reality of health reform in New Zealand", Social Science \& Medicine, $\mathrm{n}^{\circ}$ 61, 2005, p. 253-262.

- Baru R.V., Private Healthcare in India: Social Characteristics and Trends, New Delhi, Sage, 1998.

- BarU R.V., "Commercialization and the public sector in India: implications for values and aspirations", in M. MACKINTOSH et M. Koivusalo (éds), Commercialization of Health Care. Global and Local Dynamics and Policy Responses, Londres, Palgrave Macmillan, 2005, p. 101-116.

- Berman P. et M.E. Khan (éds), Paying for India's Health Care, New Delhi, Sage, 1993.

- Government of India, National Health Policy, New Delhi, Government of India, 1984.

- JefFery R., «Health planning in India 1951-1984 : the role of the Planning Commission", Health Policy and Planning, vol. 1, $\mathrm{n}^{\circ} 2,1986$, p. 127-137.

- Jeffery R., The Politics of Health in India, Berkeley, University of California Press, 1988.

- Kamat V.R., «Private practitioners and their role in the resurgence of malaria in Mumbai (Bombay) and Navi Mumbai (New Bombay), India: serving the affected or aiding an epidemic?", Social Science \& Medicine, vol. 52, $\mathrm{n}^{\circ} 6,2001$, p. 885-909.

- Metboul M., «L'introuvable césure publicprivé en Algérie. De nouveaux objets en santé dans le champs de la privatisation des soins", Autrepart, n²9, 2004, p. 13-28.

- Ministry of Health and Family Welfare, National Health Policy, New Delhi, Government of India, 2002.

- Ministry of Health and Family Welfare, Annual Report 2002-2003, New Delhi, Government of India, 2003.

- Miranda E., J. L. Scarpaci et I. Irarràzaval, "A decade of HMOs in Chile : market behavior, consumer choice and the state», Health \& Place, vol. $1, \mathrm{n}^{\circ} 1,1995$, p. 51-59.
- Mohan J., «Explaining geographies of health care: a critique», Health and Place, $\mathrm{n}^{\circ} 3,1998$, p. 113-124.

- Mohan J., Planning, Markets and Hospitals, Londres, Routledge, 2002.

- MuraleEdharan V. R., "Rural health care in Madras Presidency : 1919-1939", Indian Economic \& Social History Review, $\mathrm{n}^{\circ} 24,1987$, p. 323-334.

- National Commission on Macroeconomics AND Health, Financing and Delivery of Health Care Services, New Delhi, Government of India, 2005.

- National Statistical Survey Organisation, Socio-Economic Survey, 42nd Round, New Delhi, Government of India, 1987.

- National Statistical Survey Organisation, Socio-Economic Survey, 52nd Round, New Delhi, Government of India, 1996.

- National Statistical Survey Organisation, Socio-Economic Survey, 60th Round, New Delhi, Government of India, 2004.

- Omvedt G., «Capitalist agriculture and rural classes in India ", Economic and Political Weekly, vol. 16, n52, 1981, p. 140-159.

- Public Account Committee, Allotment of Land to Private Hospitals and Dispensaries by Delhi Development Authority, New Delhi, Lok Sabha, 2005.

- Purohit B., «Private initiatives and policy options : recent health system experience in India", Health Policy and Planning, vol.16, $\mathrm{n}^{\circ} 1,2001$, p. 87-97.

- Rao M. (éd.), Disinvesting in Health. The World Bank's Prescriptions for Health, New Delhi, Sage, 1999.

- RAYmer S., "Indian doctors help fill US health care needs", Yale Global, 16 février 2004.

- Sevalraju V. et al., Trends in Public Spending on Health in India, New Delhi, National Institute of Public Finance and Policy, 2001.

- Starr P., The Social Transformation of American Medicine, New York, Basic Books, 1982. 


\section{Bertrand Lefebvre De la planification au marché}

- Tuohy C. J., Accidental Logics. The Dynamics of Change in the Health Care Arena in the United States, Britain, and Canada, New York, Oxford University Press, 1999.

- United Nations Development Programme, Human Development Report, New York, Oxford University Press, 2004.

- World BANK, World Development Report: Investing in health, Washington, Oxford University Press, 1993.
- Yamey G., «World Bank funds private hospitals in India», British Medical Journal, vol.32, 3 février 2001.

- Yesudian C.A.K., «Behaviour of the private sector in the health market of Bombay", Health Policy and Planning, vol. 9, $\mathrm{n}^{\circ}$ 1, 1994, p. $72-80$. 